\title{
P03-6-8 Poster session
}

\section{Barley b-glucan improves metabolic condition via short-chain fatty acids produced by gut microbial fermentation}

\author{
Keita Watanabe, Satsuki Taira, Junki Miyamoto, Ikuo Kimura \\ Tokyo University of Agriculture and Technology Graduate School of Agriculture, Japan
}

Dietary barley b-glucan (BG) is believed to have metabolic benefits. However, the underlying mechanisms remain less well understood due to the reported inconsistencies in the effects of BG on satiety, energy intake, weight loss, and glycemic control. The objective of this study was to clarify the molecular mechanism underlying the metabolic benefits of barley BG using a mouse model of high fat diet (HFD)-induced obesity. Male 4-wk-old C57BL/6J mice were fed an HFD with 20\% barley flour that contains either high level of BG (HBG) or low level of BG (LBG) under both conventional and germ-free (GF) conditions for 12 consecutive weeks. In addition, mice were fed either an HFD with 5\% cellulose (HFC) or 5\% barley BG (HFB) for 12 wks. Then, metabolic parameters, gut microbial compositions, and the production of fecal short-chain fatty acids (SCFAs) were analyzed. A significant decrease in overall weight gain and fat mass was observed in HBG-fed mice as compared to the controls at 16 weeks of age. The secretion of gut hormones PYY and GLP-1 were also augmented in HBG-fed mice, thereby reducing food intake and improving insulin sensitivity by restructuring the gut microbiota and enhancing the production of SCFAs. These effects in HBG-fed mice were abolished under GF conditions. Moreover, the HFB diets also increased PYY and GLP-1 secretion, and decreased food intake compared with that in HFC-fed mice. These results suggest that the beneficial metabolic effects of barley BG are primary due to the suppression of appetite and improvement of insulin sensitivity, which are induced by gut hormone secretion promoted via gut microbiota-produced SCFAs. 